\title{
Nivel de motivación de los estudiantes de enfermería, Chachapoyas, Perú. 2019
}

\section{Motivation level of nursing students, Chachapoyas, Peru. 2019}

\author{
Rocío Ortiz Apaestegui
}

\section{RESUMEN}

El objetivo de la investigación fue determinar el nivel de motivación para el estudio de los estudiantes de enfermería de la Universidad Nacional Toribio Rodríguez de Mendoza de Amazonas, Chachapoyas, 2019. La muestra estuvo conformada por 42 estudiantes. Los datos se recolectaron mediante un cuestionario de motivación. Los resultados indicaron que del 100\% (42) de los estudiantes de enfermería, el $85.7 \%$ (36) se encuentran poco motivados para el estudio, el 9.55\% (4) motivados y el 4.8\% (2) desmotivados. Así mismo en la dimensión de variedad el 69\% (29) se muestran poco motivados para el estudio, el 16.7\% (7) motivado y el 14.3\% (6) desmotivado. En la dimensión identidad con las tareas el 59.5\% (25) poco motivados, el 38.1\% (16) y el 2.4\% (1) motivado. En la dimensión de importancia de las tareas el 59.5\%(25) poco motivado, el 33.3\%(14) desmotivados y el 7.1\%(3) motivado; en la dimensión de autonomía en la realización de tareas el 66.7\%(28) poco motivados el $31 \%$ (13) desmotivados y el 2.4\%(1) desmotivados y en la dimensión de retroalimentación el 78.6\% (33) poco motivados, el 14.3\%(6) desmotivados y el 7.1\%(3) motivados. Se concluye que más de las tres cuartas partes de los estudiantes encuentran poco motivados para el estudio y sólo el $10 \%$ se muestran motivados.

Palabras clave: Motivación, estudio, estudiantes, enfermería.

\section{ABSTRACT}

The objective of the research was to determine the level of motivation for the study of the nursing students of the National University Toribio Rodríguez de Mendoza de Amazonas, Chachapoyas, 2019. The sample consisted of 42 students. Data were collected through a motivation questionnaire. The results indicated that of $100 \%$ (42) of the nursing students, $85.7 \%$ (36) were not very motivated for the study, 9.55\% (4) were motivated and $4.8 \%(2)$ were unmotivated. Likewise, in the dimension of variety, 69\% (29) are not very motivated for the study, 16.7\% (7) are motivated and $14.3 \%$ (6) are unmotivated. In the dimension identity with the tasks, 59.5\% (25) little motivated, $38.1 \%$ (16) and 2.4\% (1) motivated. In the dimension of importance of the tasks, $59.5 \%$ (25) little motivated, $33.3 \%$ (14) unmotivated and 7.1\% (3) motivated; In the dimension of autonomy in carrying out tasks, 66.7\% (28) were not very motivated, 31\% (13) were unmotivated and $2.4 \%$ (1) were unmotivated, and in the feedback dimension, 78.6\% (33) were not very motivated, $14.3 \%$ (6) unmotivated and 7.1\% (3) motivated. It is concluded that more than three quarters of the students find little motivated to study and only $10 \%$ are motivated.

Keywords: Motivation, study, students, nursing.

${ }^{1}$ Bach: en Enfermería-Ciencias de la Salud, Universidad Nacional Toribio Rodríguez de Mendoza de Amazonas. chiyto_ortiz@hotmail.com 


\section{INTRODUCCIÓN}

A nivel mundial, la transformación más importante de las últimas décadas ha consistido en un cambio de definición respecto de la educación universitaria: ahora se lo ve como parte constitutiva de la educación fundamental que todo ciudadano debiera poseer, y ya no como una situación excepcional o de privilegio. Así, la educación universitaria debiera considerarse como un derecho de los jóvenes y la población, perspectiva que implica un compromiso por universalizar el acceso a la educación. La motivación no es un proceso unitario, sino que abarca componentes muy diversos difíciles de relacionar e integrar de acuerdo con las múltiples teorías que han aparecido sobre el tema. Sin embargo, se da una gran coincidencia en definir a la motivación como el conjunto de procesos implicados en la activación, dirección y persistencia de la conducta. (Agudelo, 2016, p. 23).

Debido a este carácter complejo y difuso de la motivación, es necesario tener en cuenta la relación que tiene con otros conceptos referidos a la intencionalidad de la conducta como son el interés, atención selectiva dentro del campo; la necesidad, entendida como falta o carencia de algo que puede ser suministrado por una determinada actividad; el valor, orientación a la meta o metas centrales en la vida de un sujeto; y la aspiración, la expectativa de alcanzar un nivel determinado de logro. (Alonso \& Montero, 2015, p. 21).

Es evidente pues, que la motivación es la clave desencadenante de los factores que incitan el aprendizaje y, por lo tanto, es clara la relación que existe entre ellos. La asociación significativa, de magnitud variable, entre motivación y rendimiento se pone de manifiesto en los estudios que sobre ésta temática se han realizado. Al ser la motivación una variable dinámica, está sujeta a cambios en la intensidad de su relación con el rendimiento cuando interactúa con una serie de condiciones y estímulos ambientales. (Angulo, 2018, p.9).

El papel del docente es fundamental en la formación y cambio del autoconcepto académico y social de los estudiantes. El docente es la persona más influyente dentro del aula por tanto el alumno valora mucho sus opiniones y el trato que recibe. Los docentes, como parte primordial del proceso enseñanza-aprendizaje, necesitan conocer el nivel de motivación de sus estudiantes, para poder intervenir de manera efectiva en la formación intelectual de los educandos y en la creación de valores científicos, humanísticos, morales y éticos indispensables para el desarrollo integral como profesionales. (Barberá, 2010, p. 27).
La motivación hacia el estudio es el estado interno que nos anima a actuar, nos dirige en determinadas direcciones y nos mantiene en algunas actividades; es el ingrediente esencial que hace que seamos capaces de aprender algo, además la motivación es la responsable de que continuemos aprendiendo ya que los procesos cognitivos necesarios para el aprendizaje son voluntarios y están bajo el control de la persona. Es necesario conocer los motivos que impulsan al alumno a mejorar su rendimiento académico y de esta manera poder desarrollar una metodología capaz de mejorar el rendimiento del alumno tanto en el ambiente de aula como en el producto final. (Candela, 2014, p. 24).

La educación universitaria se expandió levemente en Latinoamérica ya que durante la década pasada se reportó una tasa neta de matrícula promedio que aumentó de $90 \%$ a $100 \%$. Se han llevado a cabo diferentes estudios con la finalidad de reducir la deserción escolar que se produce por el deficiente rendimiento en sus asignaturas. Así mismo se afirma que el gasto educativo en países como "Cuba, Honduras, Bolivia, México, Portugal y Venezuela supera el 5\%" del presupuesto general de la república, es por ello que la meta principal en toda Latinoamérica es universalizar la educación en todos los niveles. (Egúsquiza, 2013, p. 32).

Esta investigación contribuirá a mejorar el desempeño de los estudiantes de la Escuela profesional de Enfermería el cuál se vea afectado por un bajo nivel de motivación para el estudio ya que se podría mejorar las situaciones que provocan descontento en sus labores académicas favoreciendo de ésta manera a mantener la matrícula y disminuir las repeticiones la cual ocasiona tanto daños familiares, a la sociedad y también al Estado, en vista del costo que representa para la educación e impide el acceso a otro estudiante.

\section{MATERIAL Y MÉTODOS}

La presente investigación fue de enfoque cuantitativo; de nivel descriptivo; de tipo: Según la intervención del investigador fue observacional; según la planificación de la toma de datos fue prospectivo; según el número de ocasiones en que se midió la variable de estudio fue transversal y según el número de variables de interés fue de análisis univariado. (Supo, 2016, pp. 2 - 19).

La población estuvo constituida por 95 estudiantes y la muestra estuvo conformado por 42 estudiantes matriculados en semestre académico 2019-I en la Escuela profesional de Enfermería. Para determinar las unidades muéstrales se utilizó el muestreo probabilístico aleatorio simple, para lo cual se colocó 
en una bolsa el total de balotas de la población y se sacó uno por uno hasta completar el total de la muestra requerida. Durante el proceso de investigación, se empleó el método Inductivo deductivo. Porque permitió realizar el análisis de lo particular a lo general. Para la recolección de datos se utilizó como instrumento el cuestionario de motivación, conformado por 23 enunciados los que fueron aplicados a los estudiantes universitarios con la finalidad de valorar la motivación. Los ítems estuvieron determinados en una escala de Lickert adaptado según Job Diagnostic Survey de Hackman y Oldham con respuestas de nunca, pocas veces, a veces, casi siempre y siempre. La medición de las variables se realizó mediante la escala ordinal, con puntuaciones como motivado, poco motivado y desmotivado. El instrumento fué un instrumento validado por Sánchez-Oliva \& García-Calvo, T. (2012) cuya validez fue de: $\mathrm{VC}=6.0139>\mathrm{VT}=$ 1.6449 con una confiabilidad por Alfa de Cronbach de: $=0.08$. El análisis de los datos fue procesado en el software SPSS versión 23, Microsoft Word y Excel. El análisis de los objetivos se analizó mediante la estadística descriptiva de frecuencias, con un nivel de significancia de $\alpha=0.05$.

\section{RESULTADOS}

Tabla 1. Nivel de motivación para el estudio de los estudiantes de enfermería.

\begin{tabular}{lll}
\multicolumn{1}{c}{ Nivel de motivación } & fi & \% \\
\hline Desmotivado & 2 & 4.8 \\
Poco motivado & 36 & 85.7 \\
Motivado & 4 & 9.5 \\
\hline Total & $\mathbf{4 2}$ & $\mathbf{1 0 0}$ \\
\hline
\end{tabular}

Tabla 2. Nivel y dimensiones de motivación para el estudio de los estudiantes de enfermería.

\begin{tabular}{|c|c|c|c|c|c|c|c|c|c|c|}
\hline \multirow{3}{*}{ Nivel de motivación } & \multicolumn{10}{|c|}{ Dimensiones de la motivación } \\
\hline & \multicolumn{2}{|c|}{ Variedad } & \multicolumn{2}{|c|}{$\begin{array}{c}\text { Identidad c/ } \\
\text { tareas }\end{array}$} & \multicolumn{2}{|c|}{$\begin{array}{c}\text { Importancia } \\
\text { de tareas }\end{array}$} & \multicolumn{2}{|c|}{$\begin{array}{c}\text { Autonomía } \\
\text { para la } \\
\text { realización de } \\
\text { tareas }\end{array}$} & \multicolumn{2}{|c|}{$\begin{array}{l}\text { Retroalimentació } \\
\text { n sobre su } \\
\text { desempeño. }\end{array}$} \\
\hline & Fi & $\%$ & fi & $\%$ & fi & $\%$ & $\mathbf{F i}$ & $\%$ & fi & $\%$ \\
\hline Desmotivado & 6 & 14.3 & 16 & 38.1 & 14 & 33.3 & 13 & 31.0 & 6 & 14.3 \\
\hline Poco motivado & 29 & 69.0 & 25 & 59.5 & 25 & 59.5 & 28 & 66.7 & 33 & 78.6 \\
\hline Motivado & 7 & 16.7 & 1 & 2.4 & 3 & 7.1 & 1 & 2.4 & 3 & 7.1 \\
\hline Total & 42 & 100 & 42 & 100 & 42 & 100 & 42 & 100 & 42 & 100 \\
\hline
\end{tabular}

Tabla 03: Nivel de motivación para el estudio de los estudiantes de enfermería, según edad.

\begin{tabular}{lcccccccc}
\hline \multirow{2}{*}{$\begin{array}{l}\text { EDAD } \\
\text { (años) }\end{array}$} & \multicolumn{9}{c}{ Poco } & \multicolumn{2}{c}{ Desmotivado } & \multicolumn{2}{c}{ motivado } & \multicolumn{2}{l}{ Motivado } & \multicolumn{2}{c}{ Total } \\
\cline { 2 - 9 } & $\mathbf{f i}$ & $\%$ & fi & $\%$ & Fi & $\%$ & fi & $\%$ \\
\hline$<20$ a & 1 & 8.3 & 10 & 83.3 & 1 & 8.3 & 12 & 100 \\
$20-24$ & 1 & 3.8 & 23 & 88.5 & 2 & 7.7 & 26 & 100 \\
$25-29$ & 0 & 0 & 2 & 66.7 & 1 & 33.3 & 3 & 100 \\
30 más & 0 & 0 & 1 & 100 & 0 & 0 & 1 & 100 \\
\hline Total & $\mathbf{2}$ & 4.8 & $\mathbf{3 6}$ & $\mathbf{8 5 . 7}$ & $\mathbf{4}$ & $\mathbf{9 . 5}$ & $\mathbf{4 2}$ & 100 \\
\hline
\end{tabular}

Tabla 04: Nivel de motivación para el estudio de los estudiantes de enfermería, según edad.

\begin{tabular}{llllllllll}
\hline & \multicolumn{3}{c}{ Desmotivado } & \multicolumn{2}{c}{$\begin{array}{c}\text { Poco } \\
\text { Sexo }\end{array}$} & \multicolumn{9}{c}{ Motivado } & Motivado & Total \\
\cline { 2 - 10 } & Fi & $\%$ & fi & $\%$ & fi & $\%$ & fi & $\%$ \\
\hline Masculino & 0 & 0 & 4 & 100 & 0 & 0 & 4 & 100 \\
Femenino & 2 & 5.3 & 32 & 84.2 & 4 & 10.5 & 38 & 100 \\
\hline Total & $\mathbf{2}$ & $\mathbf{4 . 8}$ & $\mathbf{3 6}$ & $\mathbf{8 5 . 7}$ & $\mathbf{4}$ & $\mathbf{9 . 5}$ & 42 & 100
\end{tabular}


Tabla 5. Nivel de motivación para el estudio de los estudiantes de enfermería, según ciclos de estudio.

\begin{tabular}{|c|c|c|c|c|c|c|c|c|}
\hline \multirow[t]{2}{*}{ CICLO } & \multicolumn{2}{|c|}{$\begin{array}{c}\text { Desmotiva } \\
\text { do }\end{array}$} & \multicolumn{2}{|c|}{$\begin{array}{l}\text { Poco } \\
\text { motivado }\end{array}$} & \multicolumn{2}{|c|}{ Motivado } & \multicolumn{2}{|c|}{ Total } \\
\hline & fi & $\%$ & fi & $\%$ & fi & $\%$ & fi & $\%$ \\
\hline I & 0 & 0 & 9 & 100 & 0 & 0 & 9 & 100 \\
\hline III & 2 & 24.2 & 7 & 63.6 & 2 & 24.2 & 11 & 100 \\
\hline $\mathbf{V}$ & 0 & 0 & 6 & 85.7 & 1 & 14.3 & 7 & 100 \\
\hline VII & 0 & 0 & 9 & 100 & 0 & 0 & 9 & 100 \\
\hline VIII & 0 & 0 & 5 & 83.3 & 1 & 16.7 & 6 & 100 \\
\hline Total & 2 & 4.8 & 36 & 85.7 & 4 & 9.5 & 42 & 100 \\
\hline
\end{tabular}

\section{DISCUSIÓN}

Realizando un análisis de los resultados el $85.7 \%$ de los estudiantes de la escuela profesional de enfermería se encuentran poco motivados, el 9.5\% motivados y el $4.8 \%$ desmotivados (Tabla 1), lo que significa que los estudiantes se encuentran poco motivados en la variedad, le dan una regular importancia a las tareas, tienen una regular autonomía para la realización de las tareas y también para la retroalimentación sobre su desempeño.

Así mismo, más de la mitad de los estudiantes indican que están poco motivados y desmotivados para el estudio en todas las dimensiones, como se observa en la dimensión de variedad el $69 \%$ de los estudiantes se sienten poco motivados para el estudio, el 16.7\% motivado y el 14.3\% desmotivado. En la dimensión identidad con las tareas el $59.5 \%$ se sienten poco motivados, el $38.1 \%$ y el $2.4 \%$ motivado (Tabla 2).

En la dimensión de importancia de las tareas el $59.5 \%$ se sienten poco motivado, el $33.3 \%$ desmotivados y el 7.1\% motivado; en la dimensión de autonomía en la realización de tareas el $66.7 \%$ se sienten poco motivados el $31 \%$ desmotivados y el $2.4 \%$ desmotivados y en la dimensión de retroalimentación el $78.6 \%$ se sienten poco motivados el $14.3 \%$ desmotivados y el $7.1 \%$ motivados (Tabla 2).

Los resultados del presente estudio son similares con los de Cardozo, R. (2010). En la cual en su investigación "Analizar las motivaciones para el estudio y las características socio-demográficas en los estudiantes de segundo año de la carrera en la Universidad de Carabobo(UC), sede Carabobo, Venezuela y la Universidad Autónoma de Madrid(UAM), España en el período académico
2009-2010". En la cual sus resultados también indican que los estudiantes se muestran poco motivados para el estudio seguida de desmotivados. Además existe relación entre algunas motivaciones y los aspectos socio-demográficos en las muestras, no encontrándose diferencias estadísticamente significativas en las motivaciones de los estudiantes de cada Universidad para la elección de la carrera.

En los estudios de Angulo, J. (2018). Relación de la motivación y satisfacción con la profesión elegida con el rendimiento de los estudiantes de la Facultad de Educación de la Universidad Nacional Mayor de San Marcos. Sus resultados indican que el $75 \%$ de los estudiantes tienen una alta motivación, y el 20\% una baja motivación y el 5\% una mediana motivación. Así mismo, el $40 \%$ tienen un rendimiento académico bueno, el 35\% regular y el 25\% malo. En conclusión no existe relación entre las dos variables en estudio.

En función a los grupos etáreos, se observa que los estudiantes menores de 20 años el $83.3 \%$ se siente poco motivado, el $8.3 \%$ desmotivados y motivados a la vez; en las edades de $20-24$ años el $88.5 \%$ se siente poco motivado, el $7.7 \%$ motivados y $3.8 \%$ desmotivados; de 25-29 años el $66.7 \%$ se sienten poco motivado y el $33.3 \%$ motivado; y en mayores de 30 años el 100\% poco motivado (Tabla 3 ).

Los resultados son similares con los de Villar, E. (2016). Motivación para el estudio y conducta adaptativa de los estudiantes de psicología, del mismo que sus resultados indican que el $80 \%$ de los estudiantes menores de 20 años tienen una media motivación, el $10 \%$ en edades de 20-24 una baja motivación y el $10 \%$ en la edades de 25-29 una mediana motivación y el 5\% mayores de 30 años una mediana motivación. El $60 \%$ tuvo una conducta adaptativa deficiente, el $40 \%$ eficiente. Dicho estudio tiene relación con el presente estudio en cuanto a los grupos etáreos.

Así mismo que del 100\% de los estudiantes según sexo, en el sexo masculino el $100 \%$ se sienten poco motivados, en el sexo femenino el $100 \%$ se sienten desmotivado, el $84.2 \%$ poco motivados y el $10.5 \%$ motivados; el 5.3\% desmotivados (Tabla 4).

Del mismo modo se observa que en el I ciclo el $100 \%$ se sienten poco motivado; en el III ciclo el $24.2 \%$ desmotivados, el $63.6 \%$ poco motivado y el $24.2 \%$ motivados; en $\mathrm{V}$ ciclo el $85.7 \%$ poco motivados, el $14.3 \%$ motivados; en VII el $100 \%$ poco motivados y en VIII el $83.3 \%$ poco motivados y el $16.7 \%$ motivados (Tabla 5).

Finalmente se indica que los resultados del presente 
estudio son muy diferentes a las de Grimaldo, M. (2013). En la cual sus resultados indican que el 55\% de los estudiantes del quinto al octavo ciclo tienen una alta motivación, y el $40 \%$ de los estudiantes del primer al tercer ciclo tienen baja motivación. Además tienen una alta emoción para el aprendizaje, lo cual se relaciona con el estudio en cuanto la poca motivación.

La motivación no es un proceso unitario, sino que abarca componentes muy diversos difíciles de relacionar e integrar de acuerdo con las múltiples teorías que han aparecido sobre el tema. Sin embargo, se da una gran coincidencia en definir a la motivación como el conjunto de procesos implicados en la activación, dirección y persistencia de la conducta.

En la presente investigación se evidencia una preocupante situación ya que los resultados demuestran que los estudiantes de la escuela profesional de Enfermería de la Universidad Nacional Toribio Rodríguez de Mendoza de Amazonas; en su mayoría se encuentran poco motivados. Sabemos que cada estudiante viene de distintas situaciones y ámbitos donde se desconocen los medios económicos, o la mentalidad de cada uno. Entonces sería un poco difícil determinar los motivos de una poca motivación para el estudio ya que no se conoce la situación y sus condiciones de vida de cada estudiante.

Finalmente se puede indicar que la motivación para el estudio es la clave desencadenante de los factores que incitan el aprendizaje y, por lo tanto, es clara la relación que existe entre ellos. La asociación significativa, de magnitud variable, entre motivación y rendimiento se pone de manifiesto en los estudios que sobre ésta temática se han realizado. Al ser la motivación una variable dinámica, está sujeta a cambios en la intensidad de su relación con el rendimiento cuando interactúa con una serie de condiciones y estímulos ambientales.

\section{CONCLUSIONES}

Más de las tres cuartas partes de los estudiantes encuentran poco motivados para el estudio y sólo el $10 \%$ se muestran motivados.

Más de la mitad de los estudiantes en las dimensiones de Identidad con las tareas, importancia de tareas, autonomía real y retroalimentación se muestran de poco motivados a desmotivados; mientras que en la dimensión de variedad se muestran de poco motivados a motivados.

Más del 50\% de los estudiantes entre las edades de $<20$, de $20-24$ y $25-29$ años se muestran poco motivados a motivados; mientras que los de 30 a más años el $100 \%$ se muestra poco motivado para el estudio.

El $100 \%$ del sexo masculino el sienten poco motivados, en el sexo femenino el $100 \%$ se sienten desmotivado, el $84.2 \%$ poco motivados y el $10.5 \%$ motivados; el 5.3\% desmotivados.

El $100 \%$ de los estudiantes del I y VII ciclo se muestran poco motivados para el estudio, mientras que más de las tres cuartas partes de los estudiantes del III, VII y VIII ciclo también se muestran poco motivados a motivados.

\section{REFERENCIAS BIBLIOGRAFICAS}

Agudelo, S. (2016). Motivación del Estudiante de IV Semestre de Medicina Pontificia Universidad Javeriana Facultad de Medicina. [Diplomado en Docencia Universitaria] Universidad Javeriana. Bogotá, D.C.

Alonso, J., \& Montero, I. (2015). Evaluación de la motivación en sujetos adultos: el cuestionario MAPE-3. Facultad de Psicología. Universidad Autónoma de Madrid. Cuestionarios y Test.

Angulo, J. (2018). Relación de la motivación y satisfacción con la profesión elegida con el rendimiento de los estudiantes de la Facultad de Educación de la Universidad Nacional Mayor de San Marcos. [Tesis de Magíster]. Lima-Perú.

Barberá, E. (2010). Marco conceptual e investigación de la motivación humana, Revista Española de Motivación y Emoción, 1, 23-36.

Cardozo, R. (2010). Motivación y características socio-demográficas de los estudiantes de medicina de la Universidad de Carabobo, Venezuela y de la Universidad Autónoma de Madrid, España. 2009-2010. Tesis para optar el grado de Doctor Patología existencial. Madrid. España.

Egúsquiza, O. (2013). Motivación y Nivel de Satisfacción Laboral de las Enfermeras en el Servicio de Infectología y Neumología del Instituto de Salud del Niño. Tesis para optar el grado académico de Magister en Psicología.

Grimaldo, M. (2013). Manual de motivación y emoción. Universidad de San Martín de Porres. Facultad de Ciencias de la Comunicación, Turismo y de Psicología. 
Escuela Profesional de Psicología.

Cardozo, R. (2010). Motivación y características socio-demográficas de los estudiantes de medicina de la Universidad de Carabobo, Venezuela. 2009-2010. Tesis para optar el grado de Doctor Patología existencial. Madrid. España.

Villar, E. (2016). Motivación y conducta adaptativa. La búsqueda de empleo de los titulados superiores. [Tesis Doctoral]. Bellaterra. Universidad Autónoma de Barcelona. Facultad de Psicología.

Candela, C. (2014). Motivaciones y expectativas profesionales. Análisis desde la perspectiva de género. [Tesis Doctoral] Universidad de Valencia, España. 